\title{
Photonic nanojet-enhanced nanometer-scale germanium photodiode
}

\author{
Mehdi Hasan and Jamesina J. Simpson* \\ Department of Electrical and Computer Engineering, 50 South Central Campus Drive, Room 3280, \\ University of Utah, Salt Lake City, Utah 84112, USA \\ *Corresponding author: jamesina.simpson@utah.edu
}

Received 23 April 2013; revised 25 June 2013; accepted 26 June 2013;

posted 2 July 2013 (Doc. ID 189062); published 24 July 2013

\begin{abstract}
A design challenge for photodiodes yielding both high speed and responsivity is the necessity to concentrate incident light into a subwavelength active volume region. Photonic nanojets have been reported in the literature as a means to focus an incident plane wave to a subwavelength-waist propagating beam with applications ranging from next-generation DVDs to characterizing subwavelength features within dielectric targets. In the present work, a new application of photonic nanojets is proposed, focusing electromagnetic energy into a photodiode. Three-dimensional finite-difference time-domain solutions are conducted to determine the advantages of photonic nanojet-enhanced photodiodes at near-infrared wavelengths $(1310 \mathrm{~nm})$. We find that photonic nanojets provide a factor of 26 increase in the volume-integrated electric field within the subwavelength active volume of the photodiode of size $0.0045 \mu \mathrm{m}^{3}$. Furthermore, this increase is achieved independent of the incident polarization and over a broad bandwidth. Photonic nanojets may thus serve as an attractive alternative to plasmonics for some applications. (C) 2013 Optical Society of America

OCIS codes: $\quad$ (040.0040) Detectors; (040.5160) Photodetectors.

http://dx.doi.org/10.1364/AO.52.005420
\end{abstract}

\section{Introduction}

Two primary factors limit the response time of photodiodes: (1) the transit time of photogenerated carriers to the electrode, and (2) the depletion layer capacitance of the semiconductor [1]. For surface normal photodiodes, a subwavelength active region yields a short transit time (fast response); however, this can result in a very low output or responsivity because the diffraction limit of light is about half of the illuminating wavelength, and breaking this limit is required to concentrate the incident light into a subwavelength active region [2]. To overcome the major trade-off between speed and responsivity, it is necessary to concentrate the incident light efficiently to a subwavelength absorption volume.

$1559-128 \mathrm{X} / 13 / 225420-06 \$ 15.00 / 0$

(C) 2013 Optical Society of America
In recent years, plasmonic designs have been proposed as a solution to this problem [3]. Resonant plasmonic structures lead to a 2 to 3 orders of magnitude higher near-field optical intensity than the incident intensity [2]. Examples include nanoantennas, such as dipole and bow tie, and concentric grating structures [1-ㅡㄹ. However, surface plasmon resonances are generated only over a narrow range of frequencies, leading to an enhancement only at those frequencies. Further, the plasmonic structures are typically polarization dependent $[2,4,6]$.

Here, an alternative to plasmonics is proposed that can concentrate broadband electromagnetic energy into a subwavelength volume to yield efficient broadband photodiodes with polarizationindependent enhancement. To accomplish this, we exploit a homogeneous polystyrene dielectric microsphere-generated photonic nanojet at near-infrared (NIR) wavelengths $(\lambda \sim 1310 \mathrm{~nm})$ to concentrate radiation into a subwavelength absorption volume 
of germanium $(\mathrm{Ge})$. The photonic nanojet is a subwavelength-waist, high-intensity beam that can propagate over a distance longer than $\lambda$ after emerging from the shadow-side surface of an illuminated lossless dielectric microcylinder or microsphere of diameter larger than $\lambda$ [7-9]. The photonic nanojet appears for a wide range of diameters of the microcylinder or microsphere (from $\sim 2 \lambda$ to greater than $40 \lambda$ ) if the refractive index contrast relative to the background is less than about 2:1 [9-11]. Finitedifference time-domain (FDTD) [12] predicted photonic nanojets have been experimentally observed $[13,14]$, have been previously proposed for nextgeneration DVD technology [15], and have been used for detection of ultrasubwavelength-thin dielectric features [16].

In the present work, a microsphere diameter of $5 \lambda$ $(6.5 \mu \mathrm{m})$ is used so that a maximum intensity is obtained in the vicinity of the polystyrene $(n=1.59)$ surface (where the active Ge is located) [11].

From 3D FDTD simulations, we have determined the following:

- The nanojet is capable of concentrating electromagnetic energy into a Ge element of a photodiode having an active volume of $0.0045 \mu \mathrm{m}^{3}$.

- This concentration of energy is accomplished over a wide range of frequencies and for any incident polarization.

- The resulting electric $(E)$-field intensity within the $\mathrm{Ge}$ is 2 to 3 orders of magnitude higher than the incident plane wave intensity.

- A volume integration of the $E$-field intensity over the active volume for the nanojet enhancement case is 26 times larger than the value obtained without the nanojet. This integration value provides an estimation of the photocurrent enhancement [2].

- The transit time of the carriers across the $150 \mathrm{~nm}$ long depletion layers is calculated to be about $1.5 \mathrm{ps}$, and the cutoff frequency is estimated to be over $100 \mathrm{GHz}$, assuming the drift velocity of the carriers is $\sim 1 \times 10^{5} \mathrm{~ms}^{-1}$.

- With a junction area of $150 \mathrm{~nm} \times 200 \mathrm{~nm}$, the junction capacitance is estimated to be a fairly small value of $0.035 \mathrm{fF}$ based on a simple parallel-plate model.

The above results suggest an enhanced photogeneration of carriers in the subwavelength active $\mathrm{Ge}$ region. Such a nanometer-scale Ge photodiode is a potential high-speed optical signal detector.

\section{Modeled Nanometer-Scale Germanium Photodiode Geometry}

Figure 1 illustrates the device geometry (not to scale) of the Ge nanophotodiode modeled with polystyrene dielectric microsphere in the 3D FDTD grid. The polystyrene dielectric microsphere is used to

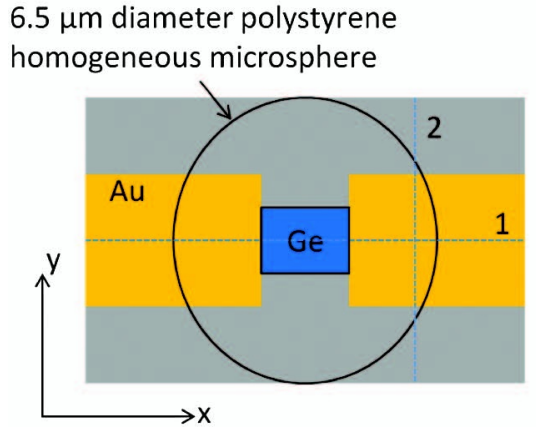

(a)

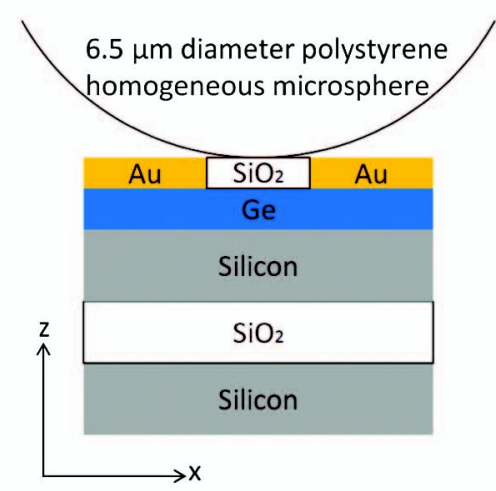

(c)

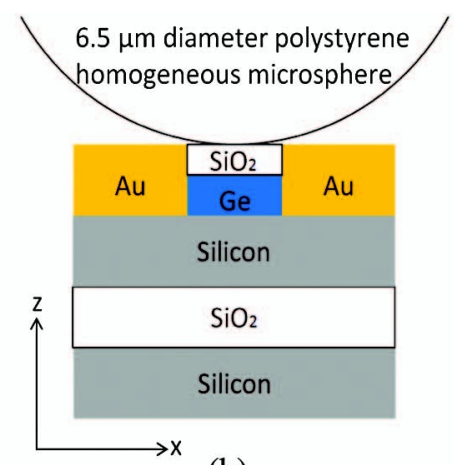

(b)

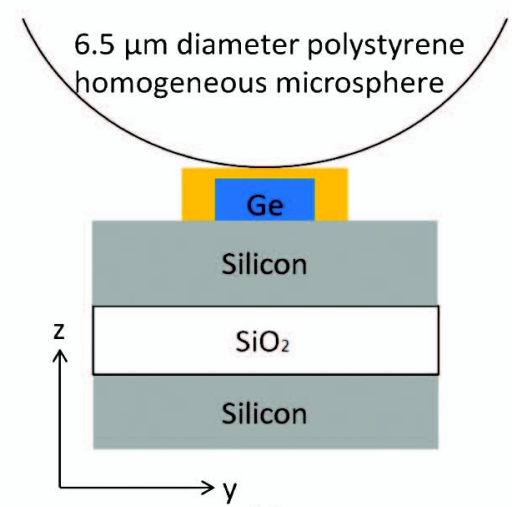

(d)

Fig. 1. Schematic of the device. (a) Top view of the device with gold electrodes in the $x$ direction. (b) Side view of the device showing Ge in the gap region between the electrodes. Two cross sections of the device are also shown as follows: (c) through line 1 in Fig. $\underline{1(a)}$ and (d) through line 2 in Fig. 1(a). 


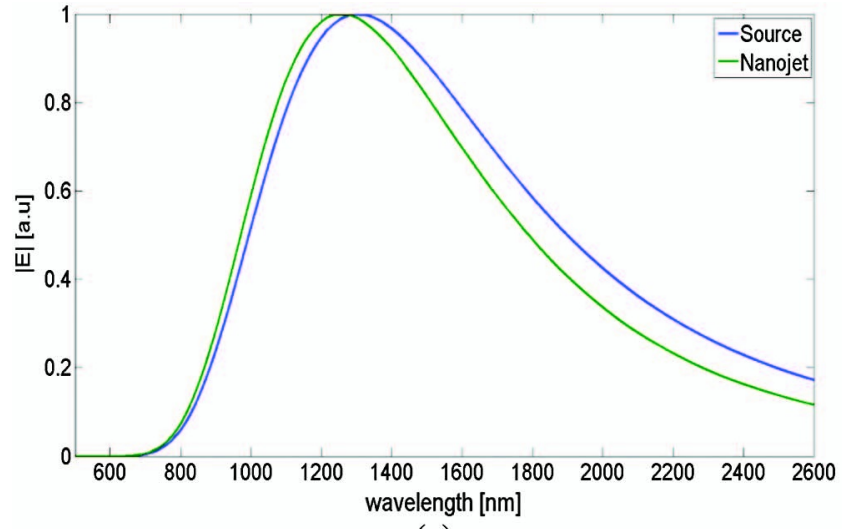

(a)

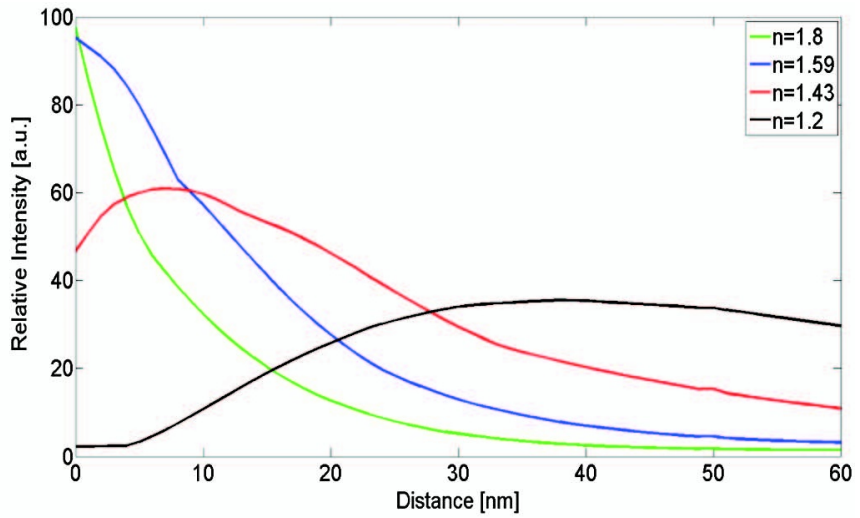

(b)

Fig. 2. (a) Comparison of the normalized spectra relative to each maxima for the source waveform (blue curve) and for the $E$-field sampled in the nanojet (green curve). (b) FDTD-computed photonic nanojet intensity relative to the incident plane wave versus distance from the microsphere's shadow-side surface along the $z$ axis. The illumination wavelength, $\lambda=1310 \mathrm{~nm}$. Four cases are shown as follows: homogenous microsphere for refractive index, $n=1.8, n=1.59$ (polystyrene), $n=1.43$ (silica), and $n=1.2$.

generate the nanojet to collect electromagnetic energy from a large area and concentrate it into the small subwavelength region of the Ge. The gold electrodes for extracting the photocurrent are also included in the model. Ge is used as the active material, which has a very high responsivity at NIR wavelengths $[17,18]$. A thick silicon $(\mathrm{Si})$ on insulator substrate is used since Ge is compatible with standard Si technology [18]. The $6.5 \mu \mathrm{m}$ diameter polystyrene (refractive index, $n=1.59$ ) microsphere is placed $50 \mathrm{~nm}$ above the Ge active layer on a $\mathrm{SiO}_{2}$ layer.

In practice, the microsphere may be covalently attached to the $\mathrm{SiO}_{2}$ layer. The Ge layer is $150 \mathrm{~nm}$ thick, and the distance between the electrodes is $150 \mathrm{~nm}$. The Ge is modeled as having a constant $n=4.35$. For $\mathrm{Si}, n=3.42$ is used, and for $\mathrm{SiO}_{2}, n=$ 1.44 is used. The gold electrodes are modeled using the Lorentz-Drude model [12,19], and the model parameters are taken from [19]. A uniform cubic grid cell size of $10 \mathrm{~nm}$ is used, and the entire modeling space spans $7.5 \mu \mathrm{m} \times 7.5 \mu \mathrm{m} \times 13 \mu \mathrm{m}$. A time step set to the Courant limit is utilized with a convolutional perfectly matched layer serving as the absorbing boundary condition [20]. The incident plane wave is $x$ polarized.

\section{Spectrum of the Nanojet and Distance of the Microsphere from the Active Layer}

Figure 2(a) shows the normalized $E$-field spectrum of the source and the polystyrene microspheregenerated nanojet. For both cases, the source is a Gaussian modulating a sinusoid with center frequency of $229 \mathrm{THz}(\lambda=1310 \mathrm{~nm})$ and falling off to $1 / e$ the maximum amplitude at 316.45 and $143.47 \mathrm{THz}(\lambda=948$ and $2091 \mathrm{~nm}$, respectively). The spectrum of the nanojet is almost identical in shape to the source spectrum (note that the small shift of the peak for the nanojet spectrum is due to the refractive index change by the microsphere). This means that the nanojet is formed over the full range of frequencies being considered, as the photonic nanojet can be generated over a wide frequency range of interest, for microspheres ranging in size from $\sim 2 \lambda$ to greater than $40 \lambda[8,9]$.

Figure 2(b) graphs the normalized intensity (relative to the incident plane wave) along the $z$ axis within the center of the nanojet as a function of distance from the microsphere's shadow-side surface for the homogeneous microsphere having diameter $5 \lambda$ $(6.5 \mu \mathrm{m})$ and a refractive index of $n=1.8, n=1.59$ (polystyrene), $n=1.43$ (silica), and $n=1.2$.

Photodetectors are square-law detectors that respond to the intensity, rather than the field amplitude of an electromagnetic wave [17]. As such, a diameter of $5 \lambda(6.5 \mu \mathrm{m})$ is used for the microsphere because a maximum intensity is obtained where the active Ge is located [in the vicinity of the polystyrene $(n=1.59)$ surface] [11]. Further results from Fig. 2(b) also support the choice of a polystyrene $(n=1.59)$ microsphere over other dielectric microspheres since it provides the maximum intensity in the vicinity of the microsphere surface (where

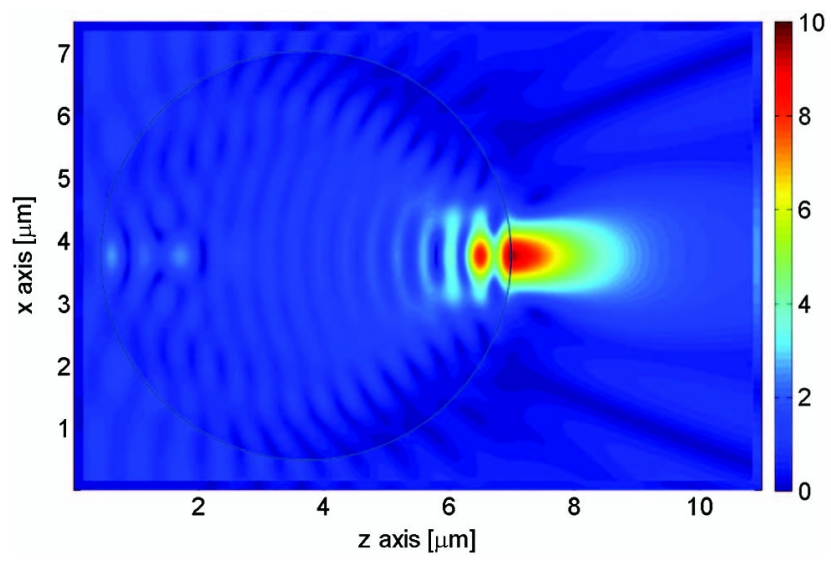

Fig. 3. Visualization of the FDTD-computed scattered $E$ field $(|E|)$ for a $6.5 \mu \mathrm{m}$ diameter polystyrene microsphere as incident wavelength, $\lambda=1310 \mathrm{~nm}$ (with no photodetector present). 
the active $\mathrm{Ge}$ is located). A thin $\mathrm{SiO}_{2}$ layer is placed between the microsphere and the photodiode that can be used to secure the microsphere in place [50 $\mathrm{nm}$ is used here, but it should be as thin as manufacturing tolerances will permit considering the amplitude for the $n=1.59$ sphere case is highest at $0 \mathrm{~nm}$, as seen in Fig. 2(b)].
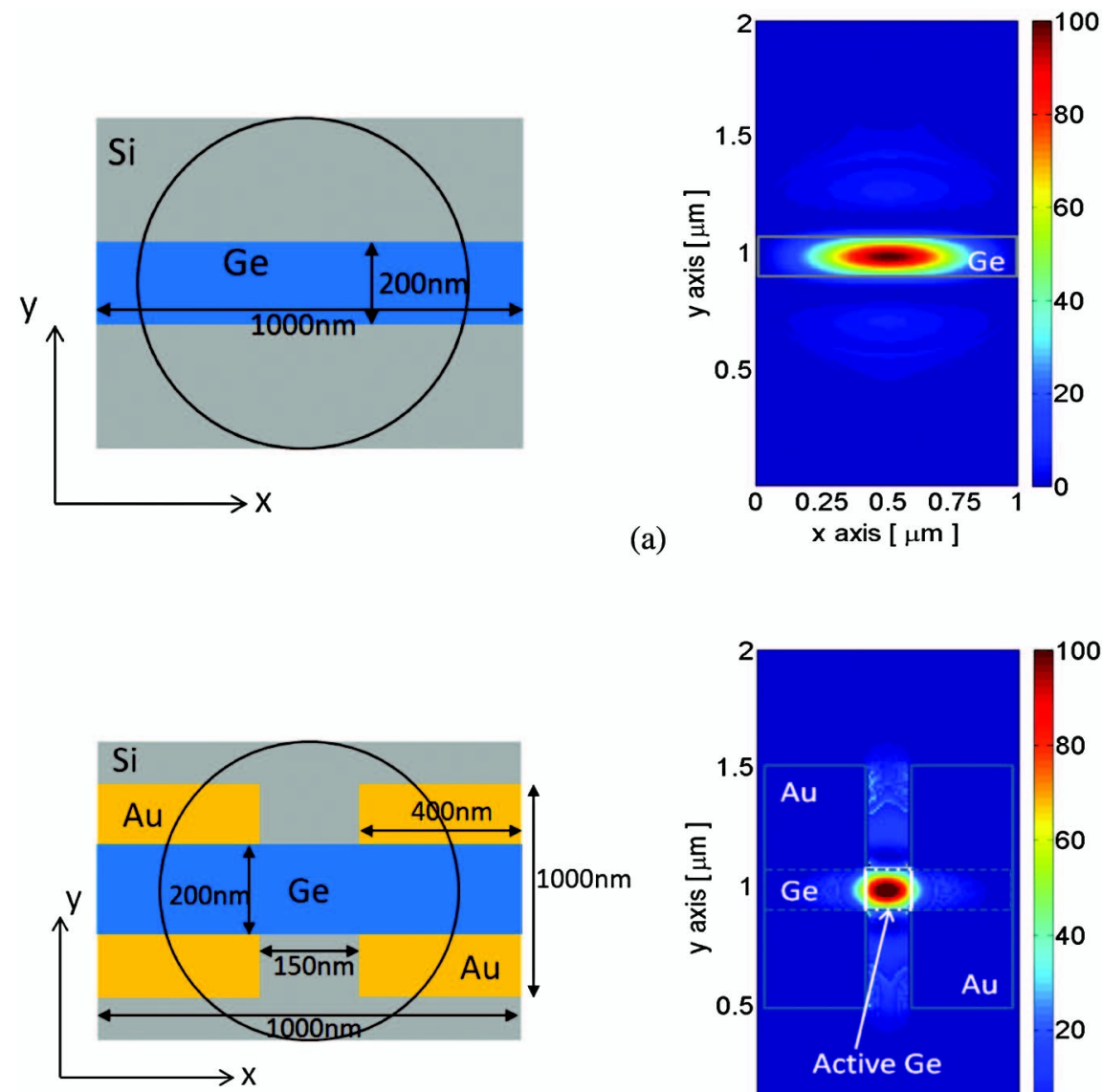

(a)

\section{Nanojet Enhancement in Nanometer-Scale Germanium Photodiode}

The homogenous polystyrene dielectric microspheregenerated nanojet operating in the NIR region $(\lambda=1310 \mathrm{~nm})$ and used to illuminate the Ge nanophotodiode is shown in Fig. 3. Figure 4 shows different planner cuts of the FDTD-calculated $E$-field (b)
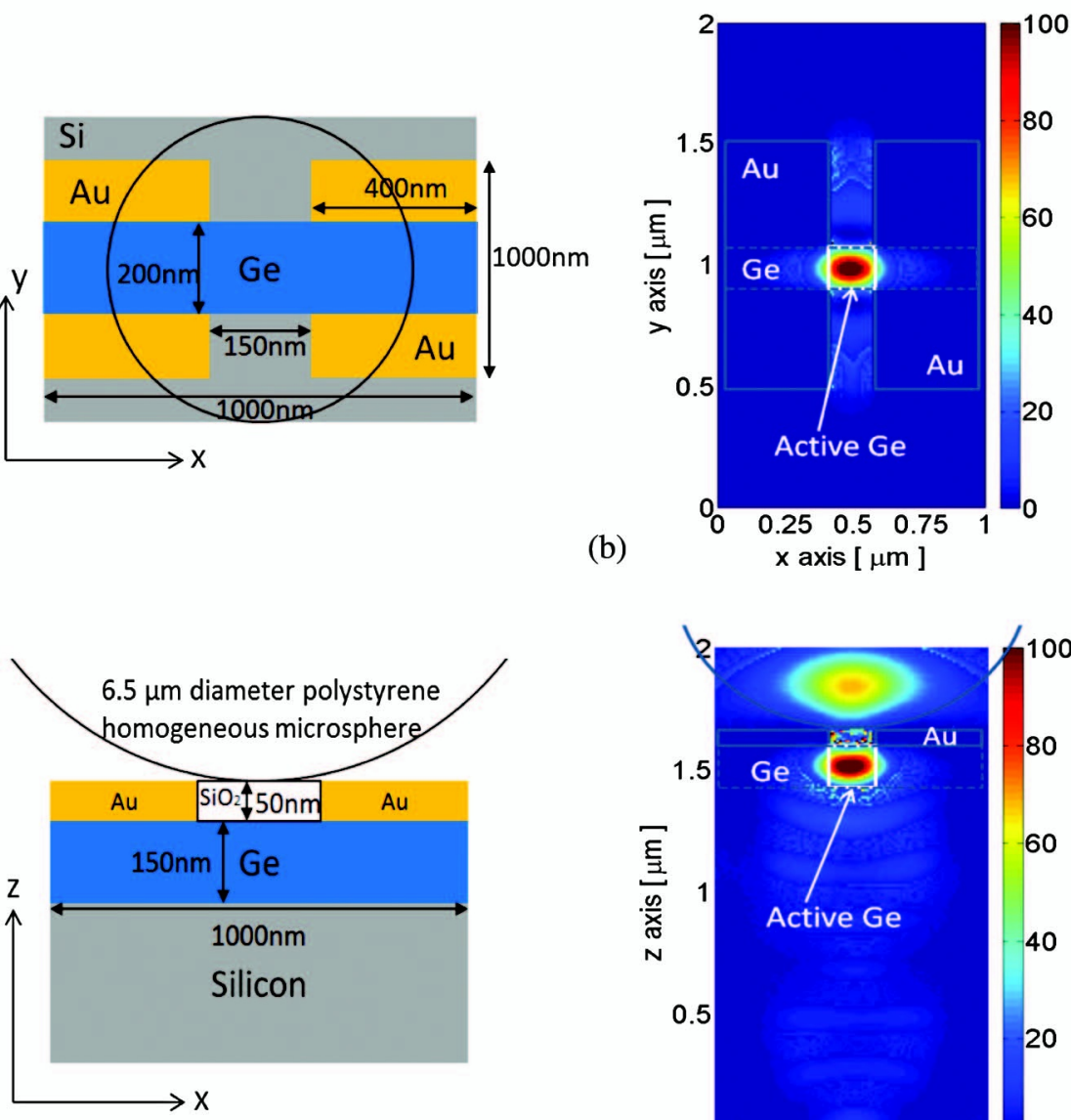

(c)

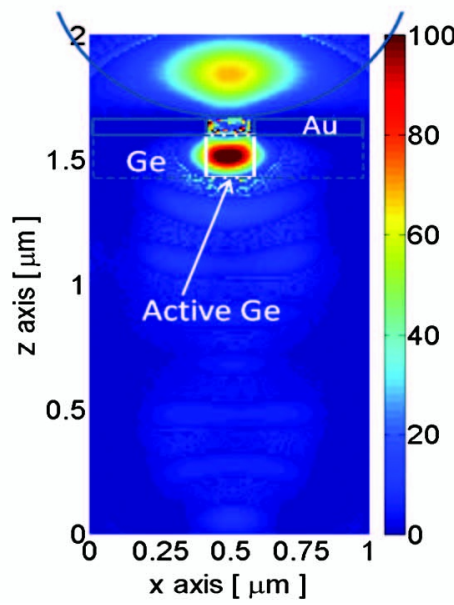

Fig. 4. FDTD-calculated optical near-field intensity $\left(|E|^{2}\right)$ in the $x-y$ plane $30 \mathrm{~nm}$ above the Si substrate, for device structure (through the Ge): (a) for the case of no gold electrodes and (b) with gold electrodes. FDTD-calculated $|E|^{2}$ for (c) the $x-z$ plane for the case with gold electrodes [through line 2 in Fig. 1(a)]. The color scale bar refers to the enhancement ratio relative to the squared incident field magnitude. 
intensity of the nanojet-illuminated Ge nanophotodiode. In Fig. 4(a), the nanojet is seen to concentrate the maximum $E$-field intensity to an area of approximately $200 \mathrm{~nm} \times 150 \mathrm{~nm}$ in the $x-y$ plane of the Ge layer. To take advantage of this concentration of high $E$-field intensity and to achieve high speed, the gold electrodes are placed $150 \mathrm{~nm}$ apart in the $x$ direction across the maximum intensity area. The FDTDcalculated $E$-field intensity for the case of the Ge with the gold electrodes is shown in Fig. 4(b). The maximum intensity for this case is concentrated over a subwavelength active area of $150 \mathrm{~nm} \times 200 \mathrm{~nm}$ in the $x-y$ plane as indicated by the white box. The absorption for this case is higher relative to that of Fig. 4(a) and the near-field intensity is concentrated just behind the aperture due to the subwavelength aperture effect by the gold electrodes [21]. For another perspective, the FDTD-calculated $E$-field intensity in Fig. 4(c) shows that the maximum intensity is concentrated over a subwavelength active area of $150 \mathrm{~nm} \times 150 \mathrm{~nm}$ in the $x-z$ plane as indicated by the white box. From the FDTD simulation results in Figs. 4(b) and 4(c), it is concluded that the polystyrene microsphere-generated nanojet concentrates electromagnetic energy into the active Ge of subwavelength volume of $0.0045 \mu \mathrm{m}^{3}$.

To estimate the photocurrent enhancement for the nanojet-photodiode case of Figs. 4(b) and 4(c), we perform a volume integration of $E$-field intensity over the $\mathrm{Ge}$ active volume [2]. The integration is performed over the $x$ component of the $E$ field, since $x$-polarized incident light is used. The volume integration of the $E$-field intensity within the Ge is found to be 26 times higher than that without the nanojet enhancement. This integration value provides an estimation of the photocurrent enhancement [2]. Further, since the microsphere is symmetric, a comparable enhancement for other polarizations is also expected.

The transit time of the carriers across the $150 \mathrm{~nm}$ long depletion layers is calculated to be about $1.5 \mathrm{ps}$, and the cutoff frequency is estimated to be over $100 \mathrm{GHz}$, assuming the drift velocity of the carriers is $\sim 1 \times 10^{5} \mathrm{~ms}^{-1}$. With a junction area of $150 \mathrm{~nm} \times$ $200 \mathrm{~nm}$, the junction capacitance is estimated to be a fairly small value of $0.035 \mathrm{fF}$ based on a simple parallel-plate model. These rough estimations suggest the possibility of very high-speed operation $[\underline{1}, \underline{2}]$.

\section{Conclusion and Future Work}

Using 3D FDTD simulations, a polystyrene microsphere of diameter $5 \lambda(6.5 \mu \mathrm{m})$ was shown to generate a photonic nanojet capable of concentrating electromagnetic energy over a broad spectrum and into a subwavelength volume of $0.0045 \mu \mathrm{m}^{3}$ active Ge. A volume integration of the optical intensity over this subwavelength active region yielded a factor of 26 improvements compared to that obtained without a nanojet. Further, the nanojet provided this improvement over a broad bandwidth, since nanojets are created for microspheres ranging in size from $\sim 2 \lambda$ to greater than $40 \lambda$. And, comparable enhancements are expected for all incident polarizations. Since the incident near-field intensity is efficiently concentrated to a subwavelength volume and the depletion layer is $150 \mathrm{~nm}$ long, these results indicate a significantly increased photocurrent and highspeed operation.

Future work includes experimentally testing the nanojet-enhanced nanometer-scale Ge photodiode for the increase in photocurrent and a measurement of the response time for the speed of the device.

The authors acknowledge the University of New Mexico's Center for Advanced Research Computing (CARC) and the University of Utah's Center for High Performance Computing (CHPC) for providing supercomputing resources.

\section{References}

1. T. Ishi, J. Fujikata, K. Makita, T. Baba, and K. Ohashi, "Si nano-photodiode with a surface plasmon antenna," Jpn. J. Appl. Phys. 44, L364-L366 (2005).

2. L. Tang, S. E. Kocabas, S. Latif, A. K. Okyay, D. Ly-Gagnon, K. C. Saraswat, and D. A. B. Miller, "Nanometre-scale germanium photodetector enhanced by a near-infrared dipole antenna," Nat. Photonics 2, 226-229 (2008).

3. G. Konstantatos and E. H. Sargent, "Nanostructured materials for photon detection," Nat. Nanotechnol. 5, 391-400 (2010).

4. L. Tang, D. A. B. Miller, A. K. Okyay, J. A. Matteo, Y. Yuen, K. C. Saraswat, and L. Hesselink, "C-shaped nanoapertureenhanced germanium photodetector," Opt. Lett. 31, 1519-1521 (2006).

5. L. Tang, S. Latif, and D. A. B. Miller, "Plasmonic device in silicon CMOS," Electron. Lett. 45, 706-708 (2009).

6. H. Fischer and O. J. F. Martin, "Engineering the optical response of plasmonic nanoantennas," Opt. Express 16 9144-9154 (2008).

7. Z. Chen, A. Taflove, and V. Backman, "Photonic nanojet enhancement of backscattering of light by nanoparticles: a potential novel visible-light ultramicroscopy technique," Opt. Express 12, 1214-1220 (2004).

8. X. Li, Z. Chen, A. Taflove, and V. Backman, "Optical analysis of nanoparticles via enhanced backscattering facilitated by 3-D photonic nanojets," Opt. Express 13, 526-533 (2005).

9. S. Kong, A. Taflove, and V. Backman, "Quasi one-dimensional light beam generated by a graded-index microsphere," Opt. Express 17, 3722-3731 (2009).

10. A. Heifetz, S. C. Kong, A. V. Sahakian, A. Taflove, and V. Backman, "Photonic nanojets," J. Comput. Theor. Nanosci. 6, 1979-1992 (2009).

11. S. Lecler, Y. Takakura, and P. Meyrueis, "Properties of a three-dimensional photonic jet," Opt. Lett. 30, 2641-2643 (2005).

12. A. Taflove and S. C. Hagness, Computational Electrodynamics: The Finite-Difference Time-Domain Method, 3rd ed. (Artech, 2005).

13. P. Ferrand, J. Wenger, A. Devilez, M. Pianta, B. Stout, N. Bonod, E. Popov, and H. Rigneault, "Direct imaging of photonic nanojets," Opt. Express 16, 6930-6940 (2008).

14. M. Kim, T. Scharf, S. Muhlig, C. Rockstuhl, and H. P. Herzig, "Engineering photonic nanojets," Opt. Express 19, 10206-10220 (2011).

15. S. C. Kong, A. Sahakian, A. Taflove, and V. Backman, "Photonic nanojet-enabled optical data storage," Opt. Express 16, 13713-13719 (2008).

16. C. Mendez-Ruiz and J. J. Simpson, "Detection of embedded ultra-subwavelength-thin dielectric features using elongated photonic nanojets," Opt. Express 18, 16805-16812 (2010). 
17. J. Liu, Photonic Devices (Cambridge University, 2005).

18. L. Colace and G. Assanto, "Germanium on silicon for near-infrared light sensing," IEEE Photon. J. 1, 69-79 (2009).

19. A. D. Rakic, A. B. Djurisic, J. M. Elazar, and M. L. Majewski, "Optical properties of metallic films for vertical-cavity optoelectronic devices," Appl. Opt. 37, 5271-5283 (1998).
20. J. A. Roden and S. D. Gedney, "Convolutional PML (CPML): an efficient FDTD implementation of the CFS-PML for arbitrary media," Microw. Opt. Technol. Lett. 27, 334-339 (2000).

21. J. S. White, G. Veronis, Z. Yu, E. S. Barnard, A. Chandran, S. Fan, and M. L. Brongersma, "Extraordinary optical absorption through subwavelength slits," Opt. Lett. 34, 686-688 (2009). 